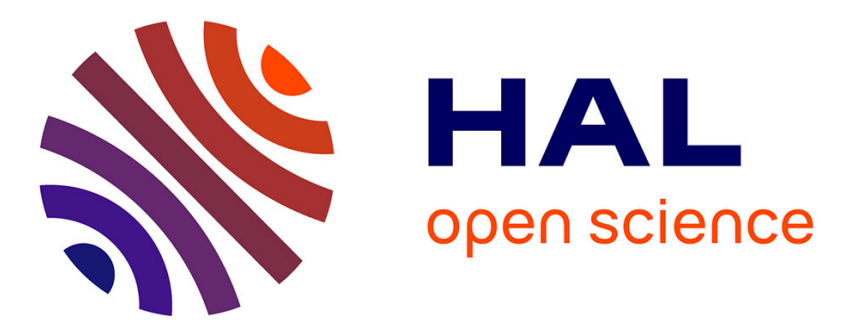

\title{
Spino-femoral muscles affect sagittal alignment and compensatory recruitment: a new look into soft tissues in adult spinal deformity
}

Hongda Bao, Bertrand Moal, Shaleen Vira, Nicolas Bronsard, Célia Amabile, Thomas Errico, Frank Schwab, Wafa Skalli, Jean Dubousset, Virginie Lafage

\section{To cite this version:}

Hongda Bao, Bertrand Moal, Shaleen Vira, Nicolas Bronsard, Célia Amabile, et al.. Spino-femoral muscles affect sagittal alignment and compensatory recruitment: a new look into soft tissues in adult spinal deformity. European Spine Journal, 2020, 29 (12), pp.2998-3005. 10.1007/s00586-020-06488-3 . hal-03106297

\section{HAL Id: hal-03106297 \\ https://hal.science/hal-03106297}

Submitted on 11 Jan 2021

HAL is a multi-disciplinary open access archive for the deposit and dissemination of scientific research documents, whether they are published or not. The documents may come from teaching and research institutions in France or abroad, or from public or private research centers.
L'archive ouverte pluridisciplinaire HAL, est destinée au dépôt et à la diffusion de documents scientifiques de niveau recherche, publiés ou non, émanant des établissements d'enseignement et de recherche français ou étrangers, des laboratoires publics ou privés. 


\title{
Spino-femoral muscles affect sagittal alignment and compensatory recruitment: a new look into soft tissues in adult spinal deformity
}

\author{
Hongda Bao ${ }^{1,6} \cdot$ Bertrand Moal $^{2} \cdot$ Shaleen Vira ${ }^{3} \cdot$ Nicolas Bronsard $^{4} \cdot$ Celia Amabile ${ }^{2}$. Thomas Errico ${ }^{3}$. \\ Frank Schwab ${ }^{1}$. Wafa Skalli ${ }^{2}$. Jean Dubousset ${ }^{5} \cdot$ Virginie Lafage $^{1}(\mathbb{C}$
}

\begin{abstract}
Objective To quantify muscle characteristics (volumes and fat infiltration) and identify their relationship to sagittal malalignment and compensatory mechanism recruitment.

Methods Female adult spinal deformity patients underwent T1-weighted MRI with a 2-point Dixon protocol from the proximal tibia up to the T12 vertebra. 3D reconstructions of 17 muscles, including extensors and flexors of spine, hip and knee, were obtained. Muscle volume standardized by bone volume and percentage of fat infiltration (Pfat) were calculated. Correlations and regressions were performed.

Results A total of 22 patients were included. Significant correlations were observed between sagittal alignment and muscle parameters. Fat infiltration of the hip and knee flexors and extensors correlated with larger C7-S1 SVA. Smaller spinal flexor/extensor volumes correlated with greater PI-LL mismatch $(r=-0.45$ and -0.51$)$. Linear regression identified volume of biceps femoris as only predictor for PT $\left(R^{2}=0.34, p=0.005\right)$ and Pfat of gluteus minimus as only predictor for SVA $\left(R^{2}=0.45, p=0.001\right)$. Sagittally malaligned patients with larger PT $\left(26.8^{\circ}\right.$ vs. $\left.17.2^{\circ}\right)$ had significantly smaller volume and larger Pfat of gluteus medius, gluteus minimus and biceps femoris, but similar values for gluteus maximus, the hip extensor. Conclusion This study is the first to quantify the relationship between degeneration of spino-femoral muscles and sagittal malalignment. This pathoanatomical study identifies the close relationship between gluteal, hamstring muscles and PT, SVA, which deepens our understanding of the underlying etiology that contributes to adult spinal deformity.
\end{abstract}

Keywords Spino-femoral muscles $\cdot$ Sagittal malalignment $\cdot$ Compensatory recruitment $\cdot$ Adult spinal deformity

Virginie Lafage

Virginie.lafage@gmail.com

1 Hospital for Special Surgery, 535 East 70th Street, New York 10021, USA

2 Arts et Metiers ParisTech, Institut de Biomécanique Humaine Georges Charpak, Paris, France

3 Hospital for Joint Diseases at NYU Langone Medical Center, New York, USA

4 Institut Universitaire de l'Appareil Locomoteur et du Sport, University Hospital of Nice, Nice, France

5 Académie Nationale de Médecine, 16 rue Bonaparte, Paris, France

6 Nanjing Drum Tower Hospital, Nanjing University, Nanjing, China

\section{Introduction}

Abnormal pelvic anatomical shape has been implicated in the development of sagittal malalignment in adult spinal deformity (ASD), as does the lack of compensatory mechanism recruitment [1]. However, muscles have been an under-appreciated component that cannot be overlooked in the investigation of ASD. Prior studies on lumbar degenerative kyphosis reveal that kyphotic patients have significantly smaller lumbar muscle and higher proportion of fat deposits in multifidus and erector spinae muscles [2]. Indeed, muscle degeneration requires more attention due to the close relationship with sagittal malalignment. Previous studies demonstrated that musculoskeletal fragility associated with sarcopenia can contribute to falls in patients since muscle performance might be altered by the loss of muscle mass and strength [3,4]. In addition to muscles located in the lumbar spine region, other parts of musculoskeletal system may 
also contribute to sagittal alignment regulation and postural control. On a higher-level point of view, postural control is a continuous process during which the neuro-central system analyzed input from the vestibular, visual and proprioceptive systems and then responds through muscular efforts.

The alteration of muscle architecture and activation in lower limbs may contribute to the maintenance of upright stance in ASD patients. The compensatory mechanisms for malalignment have been well addressed, including the role of lower limbs, with increased understanding of the contribution in the recruitment chain [5, 6]. However, the role of various muscles in this compensatory cascade remains to be investigated. A better understanding of lower limb compensation and how the muscular system affects compensation would benefit clinical evaluation and targeted therapy. Therefore, the current study aims to investigate the contribution or correlation between muscle degeneration and sagittal malalignment as well as compensatory mechanism recruitment with a novel MRI 3D reconstruction technique.

\section{Methods}

\section{Subjects}

This is a prospective pilot study on muscle profiles of ASD patients with institutional review board approval and written informed consent. Inclusion criteria were as follows: female patients older than 35 years old, meeting at least one of the following ASD radiographic criteria: coronal curve larger than $30^{\circ}$, sagittal pelvic tilt (PT) larger than $20^{\circ}$, pelvic incidence (PI) and lumbar lordosis (LL) mismatch (PI-LL) larger than $10^{\circ}$, or C7-S1 sagittal vertical axis (SVA) larger than $4 \mathrm{~cm}$ [7]. Patients with previous spinal surgery history, existing hip/thigh/knee instrumentation or presenting contraindication for MRI, were excluded from this study. Only women were recruited due to higher prevalence of ASD in women. The quality of life of the patients was evaluated by SRS-22 questionnaires and Oswestry Disability Index (ODI).

\section{Radiographic analysis}

All patients underwent standing full spine films. Radiographs were measured using validated software (SpineView, Laboratory of Biomechanics ENSAM ParisTech, France) [8], and the following sagittal radiographic parameters were obtained: PI, PI-LL, PT, SVA. After calculating the PI-LL/ PT ratio, patients were stratified based on PI-LL/PT as two groups using $40 \%$ and $60 \%$ percentile as threshold to distinguish different pelvic compensation recruitment. The PI-LL/ PT ratio stands for the level of pelvic compensation involvement in the setting of lumbosacral malalignment.

\section{MRI acquisition}

MRI was performed on a $3 \mathrm{~T}$ whole-body scanner (Magnetom Verio, Siemens Healthcare, Erlangen, Germany) using a 24-channel spine matrix coil and three 4-channel flex coils from the same vendor. The imaging protocol included a T1-weighted turbo spin echo (T1w TSE) sequence for applying the two points Dixon method (TR/ $\mathrm{TE}=829 / 15.7 \mathrm{~ms}$, acquisition matrix $=512 \times 384$, in plane resolution $=0.98 \times 0.98 \mathrm{~mm}^{2}$, slice thickness $=5 \mathrm{~mm}$, slice gap $=5 \mathrm{~mm}, \mathrm{iPat}=2,40$ slices, flip angle $=150^{\circ}$, bandwidth $=315 \mathrm{~Hz} /$ pixel, turbo factor $=3$, echo spacing $=15.7$, acquisition time $=4: 38 \mathrm{~min}$ ). Water and fat images were automatically generated by the scanner. Image volume covered the proximal tibia to the lumbar spine (until inferior endplate of T12 vertebra) and was acquired in four stages. Total acquisition time was $25 \mathrm{~min}$.

\section{D Muscle Reconstruction}

The 3D reconstruction of individual muscles, listed in Table 1, was performed using Muscl'X software (ENSAM, Laboratory of Biomechanics, Paris, France), a validated software [9] already employed in previous literature [10]. Some muscles were combined, since the low contrast made an accurate separation of the individual muscles difficult. The lumbar part of the psoas was reconstructed separately, but at a point where the distinction with the iliacus was not possible, it was then integrated into the iliacus. The external obturator, adductor longus, brevis and magnus and pectineus were reconstructed into a single group named "Adductor." The vastus lateralis and intermedius were reconstructed together. The muscle reconstructions were done on the fat images. Figure 1 (reprinted with permission from the authors of previous literature [10]) presents the 3D reconstruction of the left muscles for one patient. The femurs were also reconstructed on the water images; the contrast between the cortical and cancellous bones was greater on water images.

Right and left muscles were grouped according to the joint (spine, hip and knee) and by mechanical action ( $\frac{\text { Flexor }}{\text { Extensor }}$ of spine, hip and knee) (Table 1). The function of muscles is summarized in Table 1, while the function of gluteus medius and minimus was left empty due to their complexity.

\section{Quantification of fat and contractile components and muscle's parameters}

The fat-water ratio by voxel was calculated as previously reported [10]. From the 3D reconstructions, muscular volumes were calculated (Vmuscle) for each muscle, and the 
Table 1 Muscles reconstructed in this study and then grouped by function and joints for further analysis

\begin{tabular}{|c|c|c|c|c|c|c|c|}
\hline & $\begin{array}{l}\text { Abbreviations in } \\
\text { this article }\end{array}$ & $\begin{array}{l}\text { Spine } \\
\text { extensor }\end{array}$ & Spine flexor & Hip extensor & Hip flexor & $\begin{array}{l}\text { Knee } \\
\text { extensor }\end{array}$ & Knee flexor \\
\hline Quadratus lumborum & Q.L & $\mathrm{x}$ & - & - & - & - & - \\
\hline Erectus spinae & E.S & $\mathrm{x}$ & - & - & - & - & - \\
\hline Iliopsoas & - & - & $\mathrm{x}$ & - & $\mathrm{x}$ & - & - \\
\hline Biceps femoris short & B.F.S & - & - & $X$ & - & - & $\mathrm{x}$ \\
\hline Biceps femoris long & B.F.L & - & - & $X$ & - & - & $\mathrm{x}$ \\
\hline Semi-membranosus & S.M & - & - & $X$ & - & - & $\mathrm{x}$ \\
\hline Semi-tendinosus & S.T & - & - & $X$ & - & - & $\mathrm{x}$ \\
\hline Gluteus maximus & G.max & - & - & $X$ & - & - & - \\
\hline Rectus femoris & R.F & - & - & - & $\mathrm{x}$ & $\mathrm{x}$ & - \\
\hline Gracilis & G & - & - & - & $\mathrm{x}$ & - & $\mathrm{x}$ \\
\hline Sartorius & $\mathrm{S}$ & - & - & - & $\mathrm{x}$ & - & $\mathrm{x}$ \\
\hline Adductor & A & - & - & - & $\mathrm{x}$ & - & - \\
\hline Tensor fascia lata & T.F.L & - & - & - & $\mathrm{x}$ & - & - \\
\hline Vastus lateralis and intermedius & V.L & - & - & - & - & $\mathrm{x}$ & - \\
\hline Vastus medialis & V.M & - & - & - & - & $\mathrm{x}$ & - \\
\hline Gluteus medius & G.med & - & - & - & - & - & - \\
\hline Gluteus minimus & G.min & - & - & - & - & - & - \\
\hline
\end{tabular}

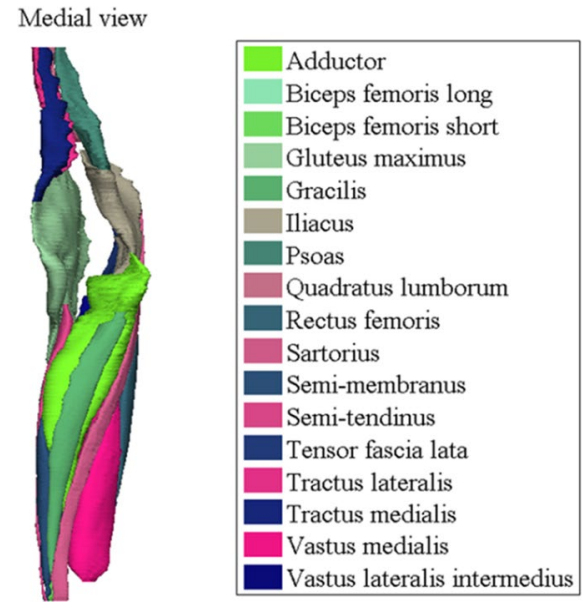

Fig. 1 Medial and frontal view of all the left muscles reconstructed for one patient. (Reprint with permission from [10])

model of repartition was used to calculate the volumes of fat components (Vfat) and contractile components (Vcont) $(\mathrm{V}$ muscle $=\mathrm{Vfat}+\mathrm{V}$ cont $)$. The volumes were then normalized between individuals based upon the volume of the right femur (Pmuscle $=$ Vmuscle/Vfemur). The fat infiltration was evaluated via the percentage of fat component (Pfat $=100 *$ Vfat/Vmuscle). Lower volume (smaller Vmuscle and Pmuscle) and more fat infiltration (larger Pfat) indicated a greater muscle degeneration.

\section{Statistical analysis}

Muscle volume and fat infiltration were characterized by mean and standard deviation. Correlations between each muscle/muscle group and radiographic parameters were performed. Stepwise linear regressions of PT and SVA using all muscle parameters were performed with stepwise at the first, and then, linear regression of PT and SVA using volume and fat infiltration of significantly related muscle was again performed. In addition, muscle parameters were compared between patients with well alignment and malalignment using nonparametric $\mathrm{t}$ test. For each statistical analysis, the level of significance was set at 0.05 .

\section{Results}

\section{Demographic}

A total of 22 ASD patients were enrolled in this study with a mean age of $61.05 \pm 12.55$ years old and mean BMI of 23.38 (Range: 20.08-32.45). All radiographic parameters are summarized in Table 2, as well as the mean fat infiltration. The spine extensor possessed the largest fat infiltration (31.73\%), followed by spine flexor $(25.80 \%)$, hip extensor (15.27\%) and knee flexor (13.14\%).

In all patients, age was positively correlated with Pfat of almost all muscle groups ( $p<0.05$, Online Appendix 1), indicating larger muscle degeneration as age increased. Meanwhile, BMI was barely correlated with volume and fat 
Table 2 Summary of radiographic characteristics and muscle of the cohort

\begin{tabular}{llrr}
\hline & $N$ & Mean & SD \\
\hline PI-LL $\left({ }^{\circ}\right)$ & 22 & 1.38 & 19.24 \\
C7-S1 SVA (mm) & 22 & 16.06 & 56.60 \\
T1PA $\left(^{\circ}\right)$ & 22 & 17.12 & 12.55 \\
SS $\left(^{\circ}\right)$ & 22 & 31.18 & 10.56 \\
PT $\left(^{\circ}\right)$ & 22 & 22.69 & 11.54 \\
PI $\left(^{\circ}\right)$ & 22 & 53.87 & 13.41 \\
T4-T12 kyphosis $\left({ }^{\circ}\right)$ & 22 & 45.04 & 18.84 \\
Thoracic Cobb angle $\left(^{\circ}\right)$ & 14 & 36.77 & 20.32 \\
Thoracolumbar/lumbar Cobb & 19 & 29.95 & 16.78 \\
$\quad$ angle $\left({ }^{\circ}\right)$ & & & \\
Fat infiltration of muscles & & & \\
Spine flexor $(\%)$ & 22 & 25.80 & 11.54 \\
Spine extensor $(\%)$ & 22 & 31.73 & 13.23 \\
Hip flexor $(\%)$ & 22 & 11.60 & 4.26 \\
Hip extensor $(\%)$ & 22 & 15.27 & 6.87 \\
Knee flexor $(\%)$ & 22 & 13.14 & 4.39 \\
Knee extensor $(\%)$ & 22 & 8.82 & 3.98 \\
\hline
\end{tabular}

infiltration of muscle groups except for the correlation with Pfat of hip extensor ( $r=0.456$, Online Appendix 1).

\section{Correlation between radiographic parameters and muscle parameters}

Significant correlations were observed between sagittal alignment radiographic parameters and those describing the muscle groups (Table 3).

For regional alignment, PI-LL mismatch correlated with a decrease in spine flexor and extensors volumes $(r=-0.445$ and $-0.508, p=0.038$ and 0.016 , respectively); no correlations were observed between PI-LL and the fat infiltration of the different muscle groups (all $p>0.05$ ). Lumbar lordosis negatively correlated with the volume and fat infiltration ratios of $\frac{\text { Spine Flexor }}{\text { Spine Extensor }}(r=-0.481$ and $-0.443, p=0.024$ and 0.039 , respectively).

For global alignment, SVA correlated with all muscle groups except spine flexors (Table 3). In terms of each specific muscle, SVA moderately to strongly correlated with an increase in fat infiltration of Hamstrings, G.Med and G.Min ( $r=0.46-0.63$, all $p<0.05$, Online Appendix 2). However, volume and fat infiltration of specific spinal muscles showed no correlation with SVA; also SVA showed no correlation to standardized muscle volumes.
Table 3 Correlations between radiographic parameters and muscle parameters

\begin{tabular}{|c|c|c|c|c|c|c|c|}
\hline & PT & PI & C7-S1 SVA & PI-LL & LL & TPA & $\mathrm{PT} / \mathrm{PI}$ \\
\hline \multicolumn{8}{|l|}{ Pmuscle } \\
\hline Spine flexor & -0.531 & -0.479 & -0.237 & -0.445 & 0.126 & -0.483 & -0.378 \\
\hline Spine extensor & -0.509 & -0.084 & -0.11 & -0.508 & 0.509 & -0.41 & -0.623 \\
\hline Hip flexor & -0.402 & -0.324 & -0.362 & -0.367 & 0.159 & -0.424 & -0.292 \\
\hline Hip extensor & -0.465 & -0.278 & -0.154 & -0.296 & 0.115 & -0.379 & -0.434 \\
\hline Knee flexor & -0.433 & -0.249 & -0.329 & -0.39 & 0.244 & -0.424 & -0.416 \\
\hline Knee extensor & -0.483 & -0.483 & -0.447 & -0.477 & 0.157 & -0.524 & -0.346 \\
\hline$\frac{\text { Spine flexor }}{\text { Spine extensor }}$ & 0.041 & -0.376 & -0.04 & 0.162 & -0.481 & 0.007 & 0.333 \\
\hline$\frac{\text { Hip flexor }}{\text { Hip extensor }}$ & 0.048 & -0.031 & -0.28 & -0.132 & 0.125 & -0.086 & 0.129 \\
\hline $\begin{array}{l}\frac{\text { Knee flexor }}{\text { Knee extensor }} \\
\text { Pfat }\end{array}$ & 0.199 & 0.362 & 0.299 & 0.214 & 0.044 & 0.278 & 0.049 \\
\hline Spine flexor & 0.124 & -0.104 & 0.285 & 0.349 & -0.476 & 0.191 & 0.36 \\
\hline Spine extensor & 0.307 & 0.447 & 0.426 & 0.278 & 0.039 & 0.382 & 0.197 \\
\hline Hip flexor & 0.147 & 0.01 & 0.443 & 0.316 & -0.349 & 0.27 & 0.308 \\
\hline Hip extensor & 0.153 & 0.117 & 0.492 & 0.267 & -0.209 & 0.306 & 0.202 \\
\hline Knee flexor & 0.217 & 0.17 & 0.545 & 0.348 & -0.259 & 0.371 & 0.263 \\
\hline Knee extensor & 0.283 & 0.205 & 0.551 & 0.347 & -0.231 & 0.424 & 0.286 \\
\hline$\frac{\text { Spine flexor }}{\text { Spine extensor }}$ & -0.145 & -0.413 & -0.147 & 0.104 & -0.443 & -0.164 & 0.105 \\
\hline$\frac{\text { Hip flexor }}{\text { Hip extensor }}$ & -0.233 & -0.273 & -0.281 & -0.181 & -0.01 & -0.302 & -0.102 \\
\hline$\frac{\text { Knee flexor }}{\text { Knee extensor }}$ & -0.319 & -0.317 & -0.265 & -0.278 & 0.064 & -0.341 & -0.187 \\
\hline
\end{tabular}

Bold values indicate significant correlations 
For compensation recruitment, PT showed moderate to strong negative correlations with the volume of spinal flexor and extensor $(r=-0.531$ and -0.509 , both $p<0.05)$, knee flexor and extensor $(r=-0.433,-0.483$, both $p<0.05)$ and hip extensor $(r=-0.465, p=0.029)$. In terms of each specific muscle, volume of muscles was significantly correlated with PT including volume of G.Med, vastus lateralis and intermedius, vastus medialis, biceps femoris long, semimembranosus, iliopsoas and erectus spinae $(r=0.42-0.58$, all $p<0.05$, Online Appendix 3).

The PT/PI ratio, evaluating percentage of pelvic compensation recruitment, demonstrated strong correlation with the volume of spine extensor $(r=-0.62, p=0.003)$, suggesting that larger PT recruitment was correlated with smaller muscle volume of spine extensors.

\section{Regression to predict SVA and PT}

Stepwise linear regression for both SVA and PT was first performed based on muscle parameters. Results revealed that muscle volume of biceps femoris long was only predictor for PT $\left(R^{2}=0.34, p=0.005\right)$ and fat infiltration of G.Min as only predictor for SVA $\left(R^{2}=0.45, p=0.001\right.$, Table 4$)$. Considering strong correlation between fat infiltration of B.F Long and of G.Med as well as between fat infiltration of B.F Long and of G.Min, both volume and fat infiltration parameters for G.Med, G.Min, and BF Long were forced to be included in the second model, increasing predictive power for PT $\left(R^{2}=0.44\right)$ and SVA $\left(R^{2}=0.61\right.$, Table 4$)$.

\section{Comparison between patients with different compensation recruitments}

As summarized in Table 5, 2 subgroups were described based on PI-LL/PT to distinguish patients with pelvic compensation recruitment. Patients with larger PT recruitment were found with statistically significant smaller volume of G.med, more fat infiltration of G.min and B.F.L. Also, this subgroup was also found with a trend of smaller volume of G. min, B.F.L and a trend of increased fat infiltration of G.med.

\section{Correlation between muscle parameters and HRQOL}

In the 19 patients with HRQOL measurement, only SRS22 self-image domain was significantly correlated with the volume of spinal extensor $(r=0.514, p=0.035)$. SRS-22 function domain showed trend of correlation to (Pfat of hip flexor)/(Pfat of hip extensor) ( $p=0.070)$, while SRS-22 mental, pain domain and total score showed no correlation to muscle parameters. Regarding ODI score, the fat infiltration of knee extensor showed trend of correlation to ODI without statistical significance ( $p=0.056$, Online Appendix 4).
Table 4 Linear regression for SVA and PT with muscle parameters

\begin{tabular}{llll}
\hline Dependent variable & Independent variables & Regression method & $R^{2}$ \\
\hline C7-S1 SVA & Pfat of gluteus minimus & Stepwise & 0.45 \\
& Volume and Pfat of biceps femoris & Enter & 0.61 \\
& $\quad$ long, gluteus medius and minimus & & \\
PT & Volume of biceps femoris long & Stepwise & 0.34 \\
& Volume and Pfat of biceps femoris & Enter & 0.44 \\
& long, gluteus medius and minimus & & \\
\hline
\end{tabular}

Table 5 Comparison between patients with different pelvic compensation

\begin{tabular}{llll}
\hline & Less PT recruitment & More PT recruitment & $p$ \\
Number & 9 & 9 & \\
\hline PI-LL & $-11.34 \pm 9.84$ & $16.65 \pm 26.12$ & $\mathbf{0 . 0 0 8}$ \\
PT & $17.18 \pm 8.03$ & $26.83 \pm 14.14$ & 0.090 \\
Standardized volume of G.max & $2.66 \pm 0.31$ & $2.71 \pm 0.69$ & 0.848 \\
Standardized volume of G.med & $1.24 \pm 0.16$ & $1.08 \pm 0.15$ & $\mathbf{0 . 0 4 0}$ \\
Standardized volume of G.min & $0.40 \pm 0.05$ & $0.35 \pm 0.05$ & 0.056 \\
Standardized volume of B.F.L & $0.65 \pm 0.11$ & $0.53 \pm 0.14$ & 0.056 \\
Pfat of G.max & $12.89 \pm 4.07$ & $20.22 \pm 10.97$ & 0.078 \\
Pfat of G.med & $9.12 \pm 3.88$ & $13.67 \pm 6.89$ & 0.104 \\
Pfat of G.min & $14.92 \pm 5.53$ & $23.77 \pm 9.28$ & $\mathbf{0 . 0 2 6}$ \\
Pfat of B.F.L & $8.74 \pm 2.57$ & $13.28 \pm 5.53$ & $\mathbf{0 . 0 4 0}$ \\
\hline
\end{tabular}

Bold values indicate significant correlations 


\section{Discussion}

In sagittal malalignment scenario, in order to maintain a erect posture, the compensatory mechanism is recruited through the involvement of muscles and the corresponding radiographic presentations such as pelvic retroversion and knee flexion are the consequences [11]. A better understanding of the role of muscles in sagittal malalignment and compensation may lead to a more thorough understanding of the underlying pathoanatomical cascade that leads to ASD and would thus represent a potential therapeutic target upstream of bony pathology that may only represent the symptoms of the underlying etiology [12]. With the use of a MRI based $3 \mathrm{D}$ reconstruction of the muscles $[9,10]$, the current study has the possibility to quantitatively evaluate the volume and fat infiltration of muscles and brings more accurate description of how the muscle impacts or correlates with sagittal malalignment.

Several previously reported studies have examined the relationship between spinal curvature and the spinal extensors with cross-sectional area (CSA), which has the limitations of the position-related change of muscle CSA and the inaccuracy to represent the volume of entire muscle $[2,13]$. The result of the current study, demonstrating that larger volume of spinal extensor and smaller fat infiltration of spinal flexor both correlated with lumbar lordosis, provided more solid evidence with 3D muscle reconstruction. Spinal muscle strength could dictate the degree of lordotic curve from a biomechanical perspective. Recent modeling suggests that a spine with large lordosis requires a greater follower load (concept proposed by Patwardhan, [14] defined as internal compressive load passing through the centers of rotation of the spinal segments) in the standing position than one with minimal lordosis [15]. Thus, increased lumbar lordosis requires larger extensor musculature and more extension strength over flexion to provide sufficient follower loads, as shown in the results that both volume and fat infiltration of $\frac{\text { Spine Flexor }}{\text { Spine Extensor }}$ correlated with LL.

The current study demonstrated that pelvic retroversion was associated with and could be predicted from gluteus medius, gluteus minimus and hamstring muscle degeneration. This is the first description regarding G. Med/Min and an interesting one since previously stronger gluteus maximus, the hip extensor, was regarded as the responsible muscle for pelvic retroversion [16]. The function of G.Med and G.Min is both difficult to determine. Anatomically, G.Med and G.Min are both primary hip abductors with rotation and flexion being possible. Instead of treating G.Med as a whole muscle, Soderberg et al. divided G.Med into 3 parts [17]. Gottschalk et al. further described that anterior and middle parts act as abductor and hip flexor [18]. Beck et al. reported that G.Min acts as a flexor and an abductor of the hip and also as either an internal or external rotator [19]. Due to the complex and ever-changing function of both G.Med and G.Min, two speculations are proposed here to serve as possible explanations for how the G.Med and G.Min affect pelvic retroversion.

One speculation is that the muscle imbalance between hip extensors and flexors leads to pelvic retroversion. In the current study, G.Max, the main hip extensor, was found to be similar between patients with and without pelvic retroversion in terms of both volume and fat infiltration. Considering the weaker G.Med and G.Min which may potentially act as hip flexor, the similar G.Max would be relatively stronger due to the imbalance between flexor and extensor in patients with more PT recruitment. The imbalance between flexor and extensors was also observed in cerebral palsy patients (significantly smaller PT) with overactivated G.Med/Min and weakened G.Max, just the opposite to the ASD patients studied here [20]. Another explanation could be the reverse pathologic cascade: pelvic retroversion would lead to possible degeneration of G.Med and G.Min. Ross et al. confirmed that more pelvic retroversion would result in acetabular anteversion and cranial version and thus result in more internal rotation [21, 22]. A recent study on hip range of motion also reported that increased pelvic retroversion may cause decreased femoral neck rotation and hip external rotation [23]. If the pelvis responded to spinal malalignment with pelvic retroversion, then femoral head tends to rotated medially spontaneously and muscles responsible for internal rotation, G.Med and G.Min, could be de-activated which in turn lead to their fatty degenerative atrophy.

In addition to gluteal muscles, the hamstring was also found to be associated with pelvic retroversion as well as sagittal global alignment. Also, it is the volume of biceps femoris long that showed negative correlation with PT. Previous studies have reported similar observations in the relationship between hamstring shortness and retroverted pelvis as well as higher trunk flexion [24]. In patients with low back pain, tight hamstring was correlated with limit hip motion [25]. Hamstring shortness was also extensively reported in spondylolisthesis patients with restricted hip flexion. The hamstring tightness in Scheuermann kyphosis was also reported and defined as a definite sign or etiological factor. Hosman et al. found that tight hamstrings were linked to limit lumbar and pelvic range of motion and risk of postoperative sagittal negative imbalance indicating overretroverted pelvis [26]. In the current study, the volume of B.F.L was correlated with and could be used to predict PT, while larger SVA was correlated with more fat infiltration of B.F.L, which implied less contribution to compensatory mechanisms such as hip extension and knee flexion.

The current study also reported the feasibility to predict sagittal malalignment with only G.Med, G.Min and B.F.L. Actually, predicting sagittal alignment from muscle 
parameters is challenging in common clinical settings that are typically not equipped to obtain the appropriate advanced imaging and subsequently analyze the data rapidly. Nevertheless, the strong influence of soft tissue alterations on sagittal alignment calls for future studies to develop targeted treatments toward muscle degeneration in the setting of sagittal malalignment. The corresponding conservative treatments toward G.Med, G.Min and B.F.L may have the potential to help maintain sagittal alignment and relieve low back pain prior surgical intervention. In addition, the evaluation of patient's soft tissue profile could also aid in the understanding of patient-specific compensatory mechanisms and thus a better surgical planning. For example, perhaps sagittal malalignment patients with tight hamstrings may require less aggressive osteotomy to prevent potential postoperative negative SVA.

The limitations of the current study mainly lie in the small sample size. Full-body radiographic evaluation with femoral version, knee varus/valgus is not available at the time of patient recruitment and should be further analyzed in the future study.

\section{Conclusion}

Using novel MRI 3D reconstruction techniques, this study is the first to quantify the relationship between degeneration of spino-femoral muscle and sagittal malalignment. This pathoanatomical study identifies the close relationship between gluteal, hamstring muscles and PT, SVA, which deepens our understanding of the underlying etiology that contributes to adult spinal deformity. It is hoped that the detailed insights gained could help to devise targeted strategies and incorporate patients' soft tissue profiles for the prevention and treatment of adult spinal deformity.

Acknowledgements The manuscript submitted does not contain information about medical device(s)/drug(s). This work received funding from Youth Fund of Natural Science Foundation of Jiangsu Province (BK20180122). This work received funding from Key Project supported by Medical Science and Technology Development Foundation, Nanjing Department of Health (YKK18092).

\section{Compliance with ethical standards}

Conflict of interest The authors declared that they have no conflict of interest.

\section{References}

1. Ferrero E, Vira S, Challier V et al. (2015) Analysis of an unexplored group of sagittal deformity patients: large sagittal malalignment but low pelvic tilt. In: ISASS. p Podium 499
2. Kang CH, Shin MJ, Kim SM et al (2007) MRI of paraspinal muscles in lumbar degenerative kyphosis patients and control patients with chronic low back pain. Clin Radiol 62:479-486. https://doi. org/10.1016/j.crad.2006.12.002

3. Helbostad JL, Sturnieks DL, Menant J et al (2010) Consequences of lower extremity and trunk muscle fatigue on balance and functional tasks in older people: a systematic literature review. BMC Geriatr 10:56. https://doi.org/10.1186/1471-2318-10-56

4. Hsu W-L, Chen C-Y, Tsauo J-Y, Yang R-S (2014) Balance control in elderly people with osteoporosis. J Formos Med Assoc 113:334-339. https://doi.org/10.1016/j.jfma.2014.02.006

5. Diebo BG, Lafage R, Ferrero E et al. (2015) Musculoskeletal compensatory mechanisms for spinal malalignment: an age-related study. In: American academy of orthopaedic surgeons (AAOS). Las Vegas, Nevada

6. Ferrero E, Liabaud B, Challier V et al (2015) Role of pelvic translation and lower-extremity compensation to maintain gravity line position in spinal deformity. J Neurosurg Spine. https:// doi.org/10.3171/2015.5.SPINE14989

7. Schwab F, Ungar B, Blondel B et al (2012) Scoliosis research society - schwab adult spinal deformity classification. Spine 37:1077-1082

8. Champain S, Benchikh K, Nogier A et al (2006) Validation of new clinical quantitative analysis software applicable in spine orthopaedic studies. Eur Spine J 15:982-991. https://doi.org/10.1007/ s00586-005-0927-1

9. Moal B, Raya JG, Jolivet E et al (2014) Validation of 3D spinopelvic muscle reconstructions based on dedicated MRI sequences for fat-water quantification. Irbm 35:119-127. https://doi. org/10.1016/j.irbm.2013.12.011

10. Moal B (2015) Volume and fat infiltration of spino-pelvic musculature in adults with spinal deformity. World J Orthop 6:727. https ://doi.org/10.5312/wjo.v6.i9.727

11. Diebo BG, Varghese JJ, Lafage R et al (2015) Sagittal alignment of the spine: what do you need to know? Clin Neurol Neurosurg 139:295-301. https://doi.org/10.1016/j.clineuro.2015.10.024

12. Schwab FJ, Diebo BG, Smith JS et al (2014) Fine-tuned surgical planning in adult spinal deformity: determining the lumbar lordosis necessary by accounting for both thoracic kyphosis and pelvic incidence. Spine J 14:S73. https://doi.org/10.1016/j.spine e.2014.08.189

13. Meakin JR, Fulford J, Seymour R et al (2013) The relationship between sagittal curvature and extensor muscle volume in the lumbar spine. J Anat 222:608-614. https://doi.org/10.1111/ joa. 12047

14. Patwardhan AG, Havey RM, Meade KP et al (1999) A follower load increases the load-carrying capacity of the lumbar spine in compression. Spine 24:1003-1009. https://doi.org/10.1097/00007 632-199905150-00014

15. Meakin JR, Aspden RM (2012) Modeling the effect of variation in sagittal curvature on the force required to produce a follower load in the lumbar spine. J Mech Med Biol 12:1250013. https:// doi.org/10.1142/S0219519412004466

16. Alvim FC, Peixoto JG, Vicente EJD et al (2010) Influences of the extensor portion of the gluteus maximus muscle on pelvic tilt before and after the performance of a fatigue protocol. Rev Bras Fisioter 14:206-213

17. Soderberg GL, Dostal WF (1978) Electromyographic study of three parts of the gluteus medius muscle during functional activities. Phys Ther 58:691-696

18. Gottschalk F, Kourosh S, Leveau B (1989) The functional anatomy of tensor fasciae latae and gluteus medius and minimus. J Anat 166:179-189. https://doi.org/10.1016/j.clinbiomech.2007.07.001

19. Beck M, Sledge JB, Gautier E et al (2000) The anatomy and function of the gluteus minimus muscle. J Bone Joint Surg Br 82:358-363. https://doi.org/10.1302/0301-620X.82B3.10356 
20. Wolf SI, Mikut R, Kranzl A, Dreher T (2014) Which functional impairments are the main contributors to pelvic anterior tilt during gait in individuals with cerebral palsy? Gait Posture 39:359-364. https://doi.org/10.1016/j.gaitpost.2013.08.014

21. Ross JR, Nepple JJ, Philippon MJ et al (2014) Effect of changes in pelvic tilt on range of motion to impingement and radiographic parameters of acetabular morphologic characteristics. Am J Sports Med 42:2402-2409. https://doi.org/10.1177/0363546514541229

22. Ross JR, Tannenbaum EP, Nepple JJ et al (2015) Functional acetabular orientation varies between supine and standing radiographs: implications for treatment of femoroacetabular impingement. Clin Orthop Relat Res 473:1267-1273. https://doi. org/10.1007/s11999-014-4104-x

23. Shimizu M, Kobayashi T, Jimbo S et al. (2016) A cohort study of adult spinal deformity and its relation with hip-knee range of motion. In: AAOS. p Podium 500

24. Carregaro RL, Gil Coury HJC (2009) Does reduced hamstring flexibility affect trunk and pelvic movement strategies during manual handling? Int J Ind Ergon 39:115-120. https://doi. org/10.1016/j.ergon.2008.05.004

25. Esola MA, McClure PW, Fitzgerald GK, Siegler S (1996) Analysis of lumbar spine and hip motion during forward bending in subjects with and without a history of low back pain. Spine 21:71-78

26. Hosman AJ, de Kleuver M, Anderson PG et al (2003) Scheuermann kyphosis: the importance of tight hamstrings in the surgical correction. Spine 28:2252-2259. https://doi.org/10.1097/01. BRS.0000085097.63326.95 